\title{
Quantitative comparisons of select cultured and uncultured microbial populations in the rumen of cattle fed different diets
}

\author{
Minseok Kim and Zhongtang Yu*
}

\begin{abstract}
Background: The number and diversity of uncultured ruminal bacterial and archaeal species revealed by 165 rRNA gene (rrs) sequences greatly exceeds that of cultured bacteria and archaea. However, the significance of uncultured microbes remains undetermined. The objective of this study was to assess the numeric importance of select uncultured bacteria and cultured bacteria and the impact of diets and microenvironments within cow rumen in a comparative manner.
\end{abstract}

Results: Liquid and adherent fractions were obtained from the rumen of Jersey cattle fed hay alone and Holstein cattle fed hay plus grain. The populations of cultured and uncultured bacteria present in each fraction were quantified using specific real-time PCR assays. The population of total bacteria was similar between fractions or diets, while total archaea was numerically higher in the hay-fed Jersey cattle than in the hay-grain-fed Holstein cattle. The population of the genus Prevotella was about one log smaller than that of total bacteria. The populations of Fibrobacter succinogenes, Ruminococcus flavefaciens, the genus Butyrivibrio, and $R$. albus was at least one log smaller than that of genus Prevotella. Four of the six uncultured bacteria quantified were as abundant as $F$. succinogenes, R. flavefaciens and the genus Butyrivibrio. In addition, the populations of several uncultured bacteria were significantly higher in the adherent fractions than in the liquid fractions. These uncultured bacteria may be associated with fiber degradation.

Conclusions: Some uncultured bacteria are as abundant as those of major cultured bacteria in the rumen. Uncultured bacteria may have important contribution to ruminal fermentation. Population dynamic studies of uncultured bacteria in a comparative manner can help reveal their ecological features and importance to rumen functions.

Keywords: $16 \mathrm{~S}$ rRNA gene, Real-time PCR, Rumen, Uncultured bacteria

\section{Background}

A complex ruminal microbiome mediates hydrolysis of polymeric feedstuffs and subsequent fermentation to volatile fatty acids (VFA) that are used as the energy source for ruminant animals [1]. Microbial biomass also constitutes the major sources of protein and B vitamins for the host animals. Being the major contributors to rumen functions, bacteria have been the focus of microbiological studies of the rumen microbiome. Cultivationbased methods were used to investigate ruminal bacteria

\footnotetext{
* Correspondence: yu.226@osu.edu

Department of Animal Sciences, The Ohio State University, 2029 Fyffe Road, Columbus, OH 43210, USA
}

until the 1980s. As a result, various cultured bacteria were identified, and their functions were determined through physiological studies of model species or strains. Once rrs sequences were used to investigate diversity of ruminal bacteria [2], it became evident that cultured ruminal bacteria represent only a small portion of the ruminal bacteriome [3,4]. Kim et al. reported that rrs sequences obtained from cultured bacteria represent only $7 \%$ of all the bacterial sequences of rumen origin [4]. More than $55 \%$ of all the bacterial sequences were assigned to groups that could not be classified into any known genus [4]. Therefore, uncultured members of the ruminal bacteriome probably play a greater role in rumen functions than the cultured peers. 
Frequencies of rrs sequences are often used to infer the abundance and population dynamics of the uncultured bacteria represented. However, PCR using universal primers is well documented to have amplification bias [5]. As such, sequence frequency does not necessarily reflect the relative abundance of the bacterium represented, or the importance or weight to rumen function. In a previous study [6], specific real-time PCR assays were developed to accurately determine the distribution and population sizes of uncultured bacteria in the rumen of sheep. Some uncultured bacteria had abundance comparable to that of several cultured bacteria that are perceived as major bacteria in the rumen. We hypothesize that this also holds true for the rumen of cattle. To test this hypothesis, real-time PCR assays were used to quantify the populations of select cultured and uncultured bacteria in the rumen of cattle fed different diets.

\section{Methods}

\section{Sample collection, fractionation and DNA extraction}

Two cannulated Jersey cattle were fed only hay composed mainly of Timothy grass (designated as $\mathrm{H}$ ), and two cannulated Holstein cattle were fed a mixture consisting of $14 \%$ alfalfa forage, $42 \%$ corn silage, $6 \%$ cottonseed, and 38\% grains (designated as C) as described previously [7]. The four cannulated cattle were fed twice daily (early morning and late afternoon) and allowed to adapt to their respective diets for more than 3 weeks prior to rumen sampling. Rumen digesta samples were collected from the four cannulated cattle approximately $6 \mathrm{~h}$ after the morning feeding. Bacteria present in the liquid (Lq) and the adherent (Ad) fractions were recovered as described previously [7]. Eight fraction samples ( 2 cattle $\times 2$ diets $\times 2$ fractions) were stored at $-80^{\circ} \mathrm{C}$ prior to DNA extraction. Metagenomic DNA was extracted from each sample as described previously [8].

\section{Real-time PCR assays}

The primers and the PCR conditions used in quantifying each target were the same as those used by Stiverson et al. [6]. End-point PCR was performed to amplify the standards for Fibrobacter succinogenes, Ruminococcus albus, and Prevotella ruminicola from the genomic DNA of respective strains using the $27 \mathrm{~F}$ (5/- AGA GTT TGA TCM TGG CTC AG-3/) and the 1525R (5/- AAG GAG GTG WTC CAR CC-3/) primers on a PTC-100 thermocycler (MJ Research, Waltham, MA). On the other hand, a composite sample of the eight metagenomic DNA samples at equal amount was used to prepare samplederived standards for total bacteria, total archaea, Butyrivibrio, Prevotella, Ruminobacter amylophilus, Ruminococcus flavefaciens, Selenomonas ruminantium, and the six uncultured bacteria by end-point PCR. The six uncultured bacterial rrs clones, Ad-C1-74-3, Lq-C2-16-3,
Lq-C2-58-2, Ad-H1-14-1, Ad-H1-75-1 and Ad-H2-90-2, were recovered from sheep fed two different diets $[6,9]$. The sample-derived standards were used to reduce bias that may result from sequence variation within total bacteria, total archaea, Butyrivibrio, or Prevotella as described previously [10]. The sample-derived standards for $R$. amylophilus, $R$. flavefaciens and S. ruminantium were used because their purified genomic DNAs were not available. These sample-derived standards were amplified from the composite DNA sample using the respective primer sets as described previously [6] and then purified using a QIAquick PCR purification kit (QIAGEN). Each standard was serially diluted and the concentration from $10^{2}$ to $10^{8} \mathrm{rrs}$ copies per reaction were used in the real-time PCR assays.

Each real-time PCR assay was conducted in three technical replicates (three PCR reactions from the same DNA template) from which the mean was calculated on a Stratagene Mx3000p machine (La Jolla, CA, USA). All the cultured and the uncultured bacteria were quantified using SYBR green I (Molecular Probes) except for total bacteria that were quantified using the TaqMan assay using the same conditions as reported by Stiverson et al. [6]. The mean was also calculated from the two biological replicates (two cattle fed the same diet) and the three technical replicates of each fraction recovered from each diet.

\section{Statistical analysis}

The abundance (rrs copies per $\mu \mathrm{g}$ metagenomic DNA) of the cultured and the uncultured bacteria were compared among the four fractions using one-way analysis of variance (ANOVA) as implemented in SAS 9.1 (SAS Institute Inc., Cary, NC). Tukey's test was used to analyze difference in bacterial abundance among the four fractions. Significance was declared at $P \leq 0.05$.

\section{Results and discussion Quantification of populations of total bacteria and total archaea}

Total bacterial populations ranging from $1.71 \times 10^{8}$ to $5.19 \times 10^{8} \mathrm{rrs}$ copies/ $\mu \mathrm{g}$ DNA did not differ between the liquid (Lq) and the adherent (Ad) fractions or between the Jersey cattle fed hay only $(\mathrm{H})$ and the Holstein cattle fed corn silage and corn (C) (Table 1). However, total bacterial populations tended $(P<0.1)$ to be higher in the $\mathrm{C}$-fed Holstein cattle than in the $\mathrm{H}$-fed Jersey. Total archaeal populations ranging from $7.22 \times 10^{2}$ to $3.16 \times 10^{4} \mathrm{rrs}$ copies/ $\mu$ g DNA did not differ among the four fractions but numerically higher in the $\mathrm{H}$-fed sheep than in the C-fed sheep (Table 1). It appears that the abundance of total archaea is affected by the amount of forage in the diet. This result corroborates the previous finding that more methane is produced by animals fed diets high in forage than by animals fed diets high in 
Table 1 Copy numbers, and relative abundance of total bacteria in each fraction*

\begin{tabular}{|c|c|c|c|c|c|}
\hline Taxon & Lq-H (Jersey) & Lq-C (Holstein) & Ad-H (Jersey) & Ad-C (Holstein) & $P$ value \\
\hline Total bacteria & $1.71 \times 10^{8} \pm 1.00 \times 10^{8}$ & $5.19 \times 10^{8} \pm 5.65 \times 10^{7}$ & $1.86 \times 10^{8} \pm 2.85 \times 10^{7}$ & $4.98 \times 10^{8} \pm 5.00 \times 10^{7}$ & 0.031 \\
\hline Total archaea & $2.62 \times 10^{4} \pm 1.87 \times 10^{4}$ & $7.22 \times 10^{2} \pm 7.60 \times 10^{1}$ & $3.16 \times 10^{4} \pm 3.75 \times 10^{3}$ & $3.43 \times 10^{3} \pm 2.07 \times 10^{3}$ & 0.183 \\
\hline \multirow[t]{2}{*}{ F. succinogenes } & $3.32 \times 10^{6} \pm 1.32 \times 10^{6}$ & $3.56 \times 10^{6} \pm 4.50 \times 10^{5}$ & $5.76 \times 10^{6} \pm 1.15 \times 10^{6}$ & $5.20 \times 10^{6} \pm 1.75 \times 10^{6}$ & 0.503 \\
\hline & $(2.27 \pm 0.56 \%)^{\mathrm{ab}}$ & $(0.68 \pm 0.01 \%)^{b}$ & $(3.09 \pm 0.14 \%)^{a}$ & $(1.09 \% \pm 0.46)^{\mathrm{ab}}$ & $(0.031)$ \\
\hline \multirow[t]{2}{*}{ R. albus } & $7.59 \times 10^{4} \pm 4.41 \times 10^{4}(b)$ & $5.31 \times 10^{5} \pm 1.90 \times 10^{4}$ (a) & $3.60 \times 10^{5} \pm 1.44 \times 10^{5}(\mathrm{ab})$ & $5.87 \times 10^{5} \pm 4.15 \times 10^{4}(\mathrm{a})$ & 0.033 \\
\hline & $(0.05 \pm 0.00 \%)$ & $(0.10 \pm 0.01 \%)$ & $(0.19 \pm 0.05 \%)$ & $(0.12 \pm 0.00 \%)$ & $(0.067)$ \\
\hline \multirow[t]{2}{*}{ R. flavefaciens } & $2.56 \times 10^{6} \pm 8.24 \times 10^{5}$ (c) & $8.27 \times 10^{6} \pm 4.31 \times 10^{5(b)}$ & $8.58 \times 10^{6} \pm 1.88 \times 10^{5}$ (b) & $2.07 \times 10^{7} \pm 4.10 \times 10^{5}$ (a) & $<0.001$ \\
\hline & $(1.86 \pm 0.61 \%)^{b}$ & $(1.60 \pm 0.09 \%)^{a}$ & $(4.71 \pm 0.62 \%)^{a}$ & $(4.20 \pm 0.34 \%)^{a}$ & $(0.020)$ \\
\hline \multirow[t]{2}{*}{ Butyrivibrio } & $1.91 \times 10^{6} \pm 8.65 \times 10^{5}$ & $3.46 \times 10^{6} \pm 7.65 \times 10^{5}$ & $3.56 \times 10^{6} \pm 6.95 \times 10^{5}$ & $3.43 \times 10^{6} \pm 1.20 \times 10^{5}$ & 0.374 \\
\hline & $(1.25 \pm 0.23 \%)$ & $(0.66 \pm 0.08 \%)$ & $(2.02 \pm 0.68 \%)$ & $(0.70 \pm 0.09 \%)$ & $(0.156)$ \\
\hline \multirow[t]{2}{*}{ P. ruminicola } & $1.94 \times 10^{5} \pm 1.58 \times 10^{5}$ (b) & $6.31 \times 10^{6} \pm 1.35 \times 10^{6}$ (a) & $9.40 \times 10^{5} \pm 1.91 \times 10^{5}$ (b) & $3.88 \times 10^{6} \pm 6.20 \times 10^{5}(\mathrm{ab})$ & 0.014 \\
\hline & $(0.09 \pm 0.04 \%)^{b}$ & $(1.26 \pm 0.40 \%)^{a}$ & $(0.50 \pm 0.03 \%)^{\mathrm{ab}}$ & $(0.78 \pm 0.05 \%)^{\mathrm{ab}}$ & (0.059) \\
\hline \multirow[t]{2}{*}{ Prevotella } & $4.40 \times 10^{7} \pm 2.96 \times 10^{7}$ (b) & $1.88 \times 10^{8} \pm 2.60 \times 10^{7}$ (a) & $5.95 \times 10^{7} \pm 5.45 \times 10^{6}$ (b) & $1.20 \times 10^{8} \pm 1.00 \times 10^{6}(a b)$ & 0.022 \\
\hline & $(23.83 \pm 3.35 \%)$ & $(37.19 \pm 9.06 \%)$ & $(33.25 \pm 8.01 \%)$ & $(24.43 \pm 2.63 \%)$ & $(0.458)$ \\
\hline \multirow[t]{2}{*}{ S. ruminantium } & $1.33 \times 10^{5} \pm 1.08 \times 10^{5}$ & $8.05 \times 10^{5} \pm 2.57 \times 10^{5}$ & $1.82 \times 10^{5} \pm 2.15 \times 10^{4}$ & $6.51 \times 10^{5} \pm 1.68 \times 10^{5}$ & 0.098 \\
\hline & $(0.06 \pm 0.03 \%)$ & $(0.16 \pm 0.07 \%)$ & $(0.10 \pm 0.00 \%)$ & $(0.13 \pm 0.02 \%)$ & $(0.394)$ \\
\hline \multirow[t]{2}{*}{ R. amylophilus } & $8.18 \times 10^{4} \pm 3.61 \times 10^{4}$ & $8.43 \times 10^{5} \pm 3.48 \times 10^{5}$ & $1.04 \times 10^{5} \pm 5.86 \times 10^{4}$ & $3.02 \times 10^{4} \pm 2.46 \times 10^{4}$ & 0.084 \\
\hline & $(0.05 \pm 0.01 \%)$ & $(0.17 \pm 0.09 \%)$ & $(0.05 \pm 0.02 \%)$ & $(0.01 \pm 0.00 \%)$ & $(0.198)$ \\
\hline \multirow[t]{2}{*}{ Ad-C1-74-3 } & $7.32 \times 10^{4} \pm 4.27 \times 10^{4}$ (b) & $1.00 \times 10^{5} \pm 2.05 \times 10^{3(b)}$ & $1.86 \times 10^{5} \pm 2.95 \times 10^{4}(\mathrm{ab})$ & $3.16 \times 10^{5} \pm 4.75 \times 10^{4}$ (a) & 0.027 \\
\hline & $(0.04 \pm 0.00 \%)$ & $(0.02 \pm 0.00 \%)$ & $(0.10 \pm 0.03 \%)$ & $(0.06 \pm 0.02 \%)$ & $(0.104)$ \\
\hline \multirow[t]{2}{*}{ Lq-C2-16-3 } & $4.55 \times 10^{5} \pm 6.05 \times 10^{4}(\mathrm{~b})$ & $9.79 \times 10^{5} \pm 4.90 \times 10^{4}(\mathrm{~b})$ & $1.42 \times 10^{6} \pm 6.50 \times 10^{4}(\mathrm{ab})$ & $3.24 \times 10^{6} \pm 7.75 \times 10^{5}$ (a) & 0.003 \\
\hline & $(0.38 \pm 0.18 \%)$ & $(0.19 \pm 0.03 \%)$ & $(0.79 \pm 0.16 \%)$ & $(0.67 \pm 0.22 \%)$ & $(0.180)$ \\
\hline \multirow[t]{2}{*}{ Lq-C2-58-2 } & $1.22 \times 10^{6} \pm 7.89 \times 10^{5}(\mathrm{~b})$ & $3.08 \times 10^{6} \pm 8.40 \times 10^{5}(\mathrm{~b})$ & $4.35 \times 10^{6} \pm 9.50 \times 10^{4}(\mathrm{~b})$ & $9.43 \times 10^{6} \pm 1.15 \times 10^{6}$ & 0.008 \\
\hline & $(0.68 \pm 0.06 \%)^{b}$ & $(0.62 \pm 0.23 \%)^{b}$ & $(2.40 \pm 0.42 \%)^{a}$ & $(1.89 \pm 0.04 \%)^{\mathrm{ab}}$ & $(0.015)$ \\
\hline \multirow[t]{2}{*}{ Ad-H1-14-1 } & $1.45 \times 10^{6} \pm 2.15 \times 10^{5}$ (b) & $2.62 \times 10^{6} \pm 2.25 \times 10^{5}(\mathrm{~b})$ & $2.53 \times 10^{6} \pm 2.95 \times 10^{5}$ (b) & $6.07 \times 10^{6} \pm 5.40 \times 10^{5}$ & 0.003 \\
\hline & $(1.18 \pm 0.57 \%)$ & $(0.51 \pm 0.01 \%)$ & $(1.37 \pm 0.05 \%)$ & $(1.22 \pm 0.01 \%)$ & $(0.288)$ \\
\hline \multirow[t]{2}{*}{ Ad-H1-75-1 } & $2.22 \times 10^{5} \pm 4.30 \times 10^{4}(\mathrm{~b})$ & $1.11 \times 10^{4} \pm 7.00 \times 10^{2(b)}$ & $8.59 \times 10^{5} \pm 2.65 \times 10^{4}$ (a) & $1.61 \times 10^{5} \pm 7.36 \times 10^{4}(\mathrm{~b})$ & 0.001 \\
\hline & $(0.18 \pm 0.08 \%)^{\mathrm{ab}}$ & $(0.00 \pm 0.00 \%)^{b}$ & $(0.48 \pm 0.09 \%)^{a}$ & $(0.03 \pm 0.02 \%)^{b}$ & $(0.015)$ \\
\hline \multirow[t]{2}{*}{$\mathrm{Ad}-\mathrm{H} 2-90-2$} & $9.26 \times 10^{5} \pm 6.73 \times 10^{5}$ & $2.03 \times 10^{6} \pm 2.50 \times 10^{5}$ & $1.77 \times 10^{6} \pm 2.60 \times 10^{5}$ & $3.72 \times 10^{6} \pm 6.50 \times 10^{5}$ & 0.067 \\
\hline & $(0.48 \pm 0.11 \%)$ & $(0.40 \pm 0.09 \%)$ & $(1.00 \pm 0.29 \%)$ & $(0.77 \pm 0.21 \%)$ & $(0.256)$ \\
\hline
\end{tabular}

*Values represent means \pm standard errors.

Note: Values in parentheses indicate relative abundance of total bacteria.

grain [11]. However, it remains to be determined if the animal breeds have any effects on rumen bacteria and archaea.

\section{Quantification of cultured bacteria}

The populations of three major cellulolytic bacteria and Butyrivibrio spp. were quantified using respective specific real-time PCR assays. The populations of $F$. succinogenes, and Butyrivibrio spp. did not differ among the four fractions irrespective of diets or animal breeds. The population of $R$. flavefaciens was significantly higher in the Ad-C fraction than in the other three fractions and significantly lower in the Lq- $\mathrm{H}$ fraction than in the other three fractions (Table 1). However, the relative abundance of $F$. succinogenes was significantly higher in the Ad-H fraction than in the Lq-C fraction (Table 1). The population of $R$. albus was significantly lower in the $\mathrm{Lq}-\mathrm{H}$ fraction than in the Lq-C and the Ad-C fractions (Table 1). Among the three cellulolytic bacteria, the populations of $F$. succinogenes $\left(1.61 \times 10^{6}\right.$ to $9.96 \times 10^{6}$ rrs copies/ug DNA) and R. flavefaciens $\left(2.56 \times 10^{6}\right.$ to $2.07 \times 10^{7} \mathrm{rrs}$ copies/ $\mu \mathrm{g}$ DNA) were more abundant than that of $R$. albus $\left(7.59 \times 10^{4}\right.$ to $\left.5.87 \times 10^{5}\right)$ in any of the fractionated samples. This result supports the previous findings that the population of F. succinogenes is higher than that of $R$. albus $[3,12,13]$. However, some studies on the rumen microbiome showed contradictory results $[6,14]$. In the two latter studies $[6,14], R$. albus was 
found to be the most predominant among the three cellulolytic species in the rumen. More studies that use the same procedures for metagenomic DNA extraction and real-time PCR assays are needed to verify the predominance of $R$. albus in the rumen in the context of diet and feeding regime. F. succinogenes is a predominant cellulolytic species in the rumen of cattle. Although real-time PCR assays showed the relatively high abundance of $F$. succinogenes, no Fibrobacteres-like rrs sequences were identified from rrs clone libraries constructed from the same rumen contents as described previously [7]. The lack of Fibrobacteres-like rrs sequences seems to be due to the poor efficiency of PCR amplification with universal primers as demonstrated previously [9]. Therefore, Fibrobacteres-specific primers are needed to account for the population of $F$. succinogenes when clone libraries, denaturing gradient gel electrophoresis (DGGE) or pyrosequencing are performed in future studies. The population of the genus Butyrivibrio was greater than $10^{6} \mathrm{rrs}$ copies/ $\mu \mathrm{g}$ DNA and did not differ among the four fractions (Table 1).

The population of the genus Prevotella ranged from $4.40 \times 10^{7}$ to $1.88 \times 10^{8} \mathrm{rrs}$ copies/ $\mu \mathrm{g}$ DNA across all the fractions and was significantly higher in the $\mathrm{Lq}-\mathrm{C}$ fraction than in the Lq-H and the Ad-H fractions (Table 1). The genus Prevotella was the most abundant among known ruminal genera, and its relative abundance ranged from $24 \%$ to $37 \%$ of total bacteria across the four fractions (Table 1). This result supports that Prevotella is the most predominant genus in the rumen $[3,4]$. The relatively high abundance of the genus Prevotella in the Ad-H fraction might suggest their involvement in fiber degradation as described previously $[15,16]$. Both Selenomonas ruminantium and Ruminobacter amylophilus did not show any significant difference among the four fractions, but $R$. amylophilus tended $(P<0.1)$ to be more abundant in the Lq-C fraction than in the Ad-C fraction (Table 1).

The population of $P$. ruminicola, the major species of genus Prevotella in the rumen, was significantly higher in the Lq-C fraction than in the Lq-H and the $\mathrm{Ad}-\mathrm{H}$ fractions (Table 1). The relative abundance of $P$. ruminicola was very low compared to that of genus Prevotella (Table 1). The low relative abundance of $P$. ruminicola is consistent with the finding of two previous studies $[3,17]$, but it does not support the majority status of this species in the rumen. This result also suggests the presence of numerous uncultured Prevotella strains [12]. Isolation and characterization of uncultured Prevotella strains would help characterize as-yet uncultured Prevotella strains in future studies.

It should be noted that the abundance of the genus Prevotella might have been overestimated because the Prevotella-"specific" primers used in this study matched numerous non-Prevotella rrs sequences when compared with 13478 sequences of rumen origin [4]. In addition to 811 Prevotella sequences, the forward primer matched 582 non-Prevotella sequences belonging to genera Paraprevotella (21 sequences), Rikenella (18 sequences), Tannerella (4 sequences), Paludibacter (4 sequences), Bacteroides (4 sequences), Barnesiella (1 sequence), Hallella (1 sequence), and unclassified Bacteroidales (298 sequences), unclassified Bacteroidetes (113 sequences), other genera of family Prevotellaceae (77 sequences), unclassified Porphyromonadaceae (40 sequences), and unclassified Clostridiales (1 sequence). The reverse primer matched 541 Prevotella sequences and 59 non-Prevotella sequences that belong to other genera of family Prevotellaceae (29 sequences), unclassified Bacteroidales (6 sequences), Paraprevotella (20 sequences), and Bacteroides (4 sequences).

The population of Prevotella might have been overestimated in other studies due to lack of specificity of the primers used. For example, the forward primers used in the study by Stiverson and Weimer and Weimer et al. $[3,17]$ matched 974 Prevotella and 1055 non-Prevotella sequences collected from the rumen. The 1055 non-Prevotella sequences were assigned to unclassified Bacteroidales (477 sequences), unclassified Bacteroidetes (168 sequences), other genera of family Prevotellaceae (140 sequences), unclassified Porphyromonadaceae (129 sequences), unclassified Clostridiales (1 sequence), Rikenella (42 sequences), Barnesiella (40 sequences), Paraprevotella (37 sequences), Hallella (11 sequences), Tannerella (4 sequences), Bacteroides (4 sequences), Paludibacter (1 sequence), and Alkaliflexus (1 sequence); while the reverse primer matched 389 Prevotella and 88 non-Prevotella sequences. The 88 nonPrevotella sequences were assigned to other genera of family Prevotellaceae (74 sequences), unclassified Bacteroidales (6 sequences), and Hallella (8 sequences). Therefore, new primers are needed to improve specific quantification of this important genus in the rumen.

\section{Quantification of uncultured bacteria}

The populations of six different uncultured bacteria were quantified using specific real-time PCR assays. Ad-C1-74-3, Lq-C2-16-3 and Lq-C2-58-2 were originally recovered from sheep fed a mixture of corn and hay, whereas Ad-H1-14-1, Ad-H1-75-1 and Ad-H2-90-2 were recovered from sheep fed hay only [6,9]. The populations of Ad-C1-74-3 and LqC2-16-3 were significantly higher in the Ad-C fraction than in the Lq-C and the Lq-H fractions (Table 1). Ad-C1-74-3 and Lq-C2-16-3 were assigned to Anaerovorax [6] and 'Unclassified Ruminococcaceae, respectively. Since Anaerovorax spp. of non-rumen origin metabolizes amino acids [18], Ad-C1-74-3 may be associated with the degradation of amino acids in the rumen. The population of Lq-C2-58-2 was significantly higher in the Ad-C fraction than in the 
other fractions (Table 1). Lq-C2-58-2 was assigned to 'Unclassified Erysipelotrichaceae'. The previous study on sheep [6] also showed that the population of Lq-C2-58-2 was the most abundant in the Ad- $\mathrm{C}$ fraction. However, the relative abundance of Lq-C2-58-2 was significantly higher in the Ad- $\mathrm{H}$ fraction than in the $\mathrm{Lq}$ fractions and accounted for more than $2 \%$ of total bacteria (Table 1). The Lq-C2-58-2 might be commonly abundant in the adherent fraction of corn-fed ruminants.

The populations of Ad-H1-14-1 and Ad-H2-90-2, which were assigned to Acetivibrio and 'Unclassified Clostridia', respectively, were about $10^{6} \mathrm{rrs}$ copies $/ \mu \mathrm{g}$ DNA. The population of Ad-H1-14-1 was significantly higher in the Ad-C than in the other three fractions, while the population of Ad-H2-90-2 did not differ among all the fractions (Table 1$)$ but tended $(P<0.1)$ to be lower in the $\mathrm{Lq}-\mathrm{H}$ fraction than in the other three fractions. However, the distribution of the populations of Ad-H1-14-1 and Ad-H2-90-2 in sheep rumen [6] were not similar to that seen in the cow rumen. Ad-H1-14-1 accounted for more than $1 \%$ of total bacteria in all the fractions except the Lq-C fraction (Table 1). Because Acetivibrio only includes cellulolytic species such as $A$. cellulolyticus and A. cellulosolvens [19,20], Ad-H1-14-1 might represent an Acetivibrio bacterium that participates in fiber degradation in the rumen. Future studies targeting Acetivibrio can help further assess the importance of this genus to cellulose degradation in the rumen. The population of Ad-H1-75-1, which was assigned to 'Unclassified Clostridiales', was significantly higher in the Ad-H fraction than in the other three fractions (Table 1). Although the previous study [6] did not show significant difference in this uncultured bacterium among fractions, the population of Ad-H1-75-1 was numerically higher in sheep fed hay alone than in sheep fed hay plus corn. Thus, Ad-H1-75-1 might be a member of the biofilm adhering to feed particles and potentially involved in fiber degradation.

In our study, two measurements (rrs copy numbers vs. relative abundance) did not always result in the same significant differences among the four fractions. This discrepancy might be attributable to variable amounts of ruminal protozoal and fungal DNA contained in the metagenomic DNA that was used to normalize rrs copy numbers. More studies will need to be performed to verify this assumption.

Host genetics can have effects on the rumen microbiome and thus cattle of the same breed are typically used in the same study. In this study two different breeds were used and the number of animals analyzed were small. Nevertheless, the preliminary results of this study based on single sampling suggest that numerous uncultured bacteria are predominant in the rumen and may play an important role in ruminal fermentation.
The function and ecological features of uncultured bacteria might be inferred from their population dynamics in cattle fed different diets. Alternatively, a reverse metagenomic approach [21,22], may be used to help isolate these uncultured bacteria. The metagenomic data recovered in previous studies of ruminal samples can be used to design selective media to grow uncultured bacteria through its metabolic reconstruction, while population data, as demonstrated in this study using specific realtime PCR, can be used to choose the dilutions as inoculum. Future studies may also determine to what extend animal breeds affect rumen microbiome.

\section{Conclusions}

The populations of uncultured bacteria can be as great as those of major cultured bacteria. These uncultured bacteria are also ubiquitous in the rumen. Uncultured bacteria may play as an important role as some of the cultured bacteria, if not more. Comparative dynamic studies of uncultured bacteria in response to dietary treatments might help further reveal their ecological niche and roles in the rumen. Isolation and characterization of as-yet uncultured bacteria in the rumen would need to be attempted to define their functions and contribution to rumen functions.

\section{Abbreviations \\ rrs: $16 \mathrm{~S}$ rRNA gene; Ad: Adherent; Lq: Liquid; $\mathrm{H}$ : Cattle fed hay alone; C: Corn silage and corn plus alfalfa hay; ANOVA: Analysis of variance.}

\section{Competing interests}

Both authors have no competing interests.

\section{Authors' contributions}

ZY and MK conducted this experiment and drafted the manuscript. ZY advised MK in performing the study and writing the manuscript for publication. All authors read and approved the final manuscript.

\section{Acknowledgments}

This study was partially supported by an OARDC award (2010-007) to Z.Y.

Received: 28 February 2012 Accepted: 3 September 2012

Published: 7 September 2012

\section{References}

1. Hobson PN, Stewart CS: Rumen Microbial Ecosystem. 2nd edition. New York: Blackie Academic and Professional; 1997.

2. Stahl DA, Flesher B, Mansfield HR, Montgomery L: Use of phylogenetically based hybridization probes for studies of ruminal microbial ecology. Appl Environ Microbiol 1988, 54:1079-1084.

3. Stevenson DM, Weimer PJ: Dominance of Prevotella and low abundance of classical ruminal bacterial species in the bovine rumen revealed by relative quantification real-time PCR. Appl Microbiol Biotechnol 2007, 75:165-174

4. Kim M, Morrison M, Yu Z: Status of the phylogenetic diversity census of ruminal microbiomes. FEMS Microbiol Ecol 2011, 76:49-63.

5. Edwards JE, McEwan NR, Travis AJ, Wallace RJ: 16S rDNA library-based analysis of ruminal bacterial diversity. Antonie Van Leeuwenhoek 2004, 86:263-281.

6. Stiverson J, Morrison M, Yu Z: Populations of select cultured and uncultured bacteria in the rumen of sheep and the effect of diets and ruminal fractions. Int J Microbiol 2011, 75:165-174. 
7. Kim M, Morrison $\mathrm{M}, \mathrm{Yu}$ Z: Phylogenetic diversity of bacterial communities in bovine rumen as affected by diets and microenvironments. Folia Microbiol 2011, 56:453-458

8. Yu Z, Morrison M: Improved extraction of PCR-quality community DNA from digesta and fecal samples. Biotechniques 2004, 36:808-812.

9. Larue R, Yu Z, Parisi VA, Egan AR, Morrison M: Novel microbial diversity adherent to plant biomass in the herbivore gastrointestinal tract, as revealed by ribosomal intergenic spacer analysis and rrs gene sequencing. Environ Microbiol 2005, 7:530-543.

10. Yu Z, Michel FC, Hansen G, Wittum T, Morrison M: Development and application of real-time PCR assays for quantification of genes encoding tetracycline resistance. Appl Environ Microbiol 2005, 71:6926-6933.

11. Janssen PH: Influence of hydrogen on rumen methane formation and fermentation balances through microbial growth kinetics and fermentation thermodynamics. Anim Feed Sci Tech 2010, 160:1-22.

12. Bekele AZ, Koike S, Kobayashi Y: Genetic diversity and diet specificity of ruminal Prevotella revealed by 165 rRNA gene-based analysis. FEMS Microbiol Lett 2010, 305:49-57.

13. Mosoni P, Chaucheyras-Durand F, Bera-Maillet C, Forano E: Quantification by real-time PCR of cellulolytic bacteria in the rumen of sheep after supplementation of a forage diet with readily fermentable carbohydrates: effect of a yeast additive. J Appl Microbiol 2007, 103:2676-2685.

14. Martin C, Millet L, Fonty G, Michalet-Doreau B: Cereal supplementation modified the fibrolytic activity but not the structure of the cellulolytic bacterial community associated with rumen solid digesta. Reprod Nutr Dev 2001, 41:413-424.

15. Dodd D, Kiyonari S, Mackie RI, Cann IKO: Functional diversity of four glycoside hydrolase family 3 enzymes from the rumen bacterium prevotella bryantii B(1)4. J Bacteriol 2010, 192:2335-2345.

16. Koike S, Yoshitani S, Kobayashi Y, Tanaka K: Phylogenetic analysis of fiberassociated rumen bacterial community and PCR detection of uncultured bacteria. FEMS Microbiol Lett 2003, 229:23-30.

17. Weimer PJ, Stevenson DM, Mertens DR, Thomas EE: Effect of monensin feeding and withdrawal on populations of individual bacterial species in the rumen of lactating dairy cows fed high-starch rations. Appl Microbiol Biotechnol 2008, 80:135-145.

18. Matthies C, Evers S, Ludwig W, Schink B: Anaerovorax odorimutans gen. nov., sp. nov., a putrescine-fermenting, strictly anaerobic bacterium. Int J Syst Evol Microbiol 2000, 50:1591-1594.

19. Jindou S, Xu Q, Kenig R, Shulman M, Shoham Y, Bayer EA, Lamed R: Novel architecture of family- 9 glycoside hydrolases identified in cellulosomal enzymes of Acetivibrio cellulolyticus and Clostridium thermocellum. FEMS Microbiol Lett 2006, 254:308-316.

20. Murray WD: Acetivibrio cellulosolvens is a synonym for Acetivibrio cellulolyticus: emendation of the genus acetivibrio. Int I Syst Bacteriol 1986, 36:314-316

21. Nichols D: Cultivation gives context to the microbial ecologist. FEMS Microbiol Ecol 2007, 60:351-357.

22. Pope PB, Smith W, Denman SE, Tringe SG, Barry K, Hugenholtz P, McSweeney CS, McHardy AC, Morrison M: Isolation of Succinivibrionaceae implicated in low methane emissions from Tammar wallabies. Science 2011, 333:646-648.

doi:10.1186/2049-1891-3-28

Cite this article as: Kim and Yu: Quantitative comparisons of select cultured and uncultured microbial populations in the rumen of cattle fed different diets. Journal of Animal Science and Biotechnology 2012 3:28.

\section{Submit your next manuscript to BioMed Central and take full advantage of:}

- Convenient online submission

- Thorough peer review

- No space constraints or color figure charges

- Immediate publication on acceptance

- Inclusion in PubMed, CAS, Scopus and Google Scholar

- Research which is freely available for redistribution

Submit your manuscript at www.biomedcentral.com/submit
C Biomed Central 